\title{
Risk factors for oxaliplatin-induced vascular pain in patients with colorectal cancer and comparison of the efficacy of preventive methods
}

\author{
Yukio Suga ${ }^{1,2^{*}}$, Nana Ikeda', Manami Maeda ${ }^{1}$, Angelina Yukiko Staub ${ }^{1}$, Tsutomu Shimada², Miwa Yonezawa ${ }^{3}$, \\ Hironori Kitade ${ }^{4}$, Hideyuki Katsura ${ }^{5}$, Morihiro Okada ${ }^{6}$, Junko Ishizaki ${ }^{1}$, Yoshimichi Sai ${ }^{2}$ and Ryo Matsushita ${ }^{1}$
}

\begin{abstract}
Background: Vascular pain is a common adverse drug reaction in colorectal cancer patients receiving peripheral venous administration of oxaliplatin. The aim of this work was to identify risk factors for vascular pain, and to examine whether currently used treatments reduce its incidence.

Methods: We conducted a multicenter retrospective study in Japanese colorectal cancer patients receiving peripheral venous administration of oxaliplatin. The effects of various treatments (administration of analgesics, addition of dexamethasone to the infusion solution for $\mathrm{pH}$ adjustment, dilution of the infusion solution, or use of hot gel for warming the injection site) on the incidence of vascular pain were assessed. Risk factors for vascular pain were identified by multiple logistic regression analysis.

Results: One hundred and ninety patients who had received an oxaliplatin-containing regimen via a peripheral venous route were analyzed. None of the preventive methods examined significantly reduced the incidence of vascular pain. BMI (BMI <22), clinical stage (I-III) and oxaliplatin dosage $\left(130 \mathrm{mg} / \mathrm{m}^{2}\right.$ versus dose reduction) were identified as independent risk factors for development of vascular pain. The incidence of oxaliplatin-induced vascular pain was significantly higher in patients who had two or more risk factors.
\end{abstract}

Conclusions: BMl, clinical stage and oxaliplatin dosage were identified as independent predictive markers for oxaliplatin-induced vascular pain. Existing treatments for vascular pain are not effective in reducing its incidence.

Keywords: Vascular pain, Oxaliplatin, Colorectal cancer, Preventive method, Risk factor

\section{Background}

Oxaliplatin (L-OHP) is a third-generation platinum analog that is mainly used for treatment of advanced colorectal cancer [1] and adjuvant chemotherapy [2]. Although peripheral neuropathy is a major adverse drug reaction in patients receiving L-OHP [3-5], mild to moderate vascular pain originating around the injection site during peripheral intravenous administration of

\footnotetext{
* Correspondence: suga@staff.kanazawa-u.ac.jp

${ }^{1}$ Department of Clinical Drug Informatics, Faculty of Pharmacy, Institute of

Medical, Pharmaceutical \& Health Science, Kanazawa University, 13-1,

Takaramachi, Kanazawa, Ishikawa 920-8641, Japan

²Department of Pharmacy, Kanazawa University Hospital, Kanazawa

University, 13-1, Takaramachi, Kanazawa, Ishikawa 920-8641, Japan

Full list of author information is available at the end of the article
}

L-OHP is also a significant problem. The mechanism of L-OHP-induced vascular pain is poorly understood. Also, risk factors and preventive methods have not yet been adequately evaluated.

Propofol has also been reported to cause vascular pain, like L-OHP [6-8]. It was reported that preadministration of fentanyl or remifentanyl (opioid analgesics) tended to reduce the incidence of propofol-induced vascular pain [6]. Several methods for preventing or relieving L-OHP-induced vascular pain have been reported in clinical studies, but only small numbers of patients were involved [9-11]. Shiotsuka et al. reported that addition of $3.3 \mathrm{mg}$ of dexamethasone to the infusion solution reduced the numerical rating scale (NRS) score of vascular 
pain induced by peripheral administration of L-OHP [10]. Hibi et al. reported that warming the injection site with a hot gel was effective for managing L-OHP-induced vascular pain [11]. Studies with larger numbers of patients are necessary to confirm and compare the efficacy of these methods.

It is important to elucidate the risk factors for adverse drug reactions in order to develop effective preventive methods. For example, the use of epirubicin liquid preparation was identified as a risk factor for phlebitis [12], and we have shown that the incidence of epirubicin-induced severe phlebitis could be significantly reduced by employing a lyophilized formulation [13]. However, the risk factors for L-OHP-induced vascular pain are not clear.

The present multicenter retrospective study was conducted in order to determine whether currently used treatments are effective to reduce the incidence of vascular pain in colorectal cancer patients receiving peripheral venous administration of L-OHP. We also investigated the risk factors for L-OHP-induced vascular pain.

\section{Methods}

\section{Patients}

The present study was conducted in accordance with the Declaration of Helsinki and its amendments, and the protocol was approved by the Ethics Committee of Kanazawa University (approval no. 1462), as well as by the ethics committees of all participating hospitals, which were Ishikawa Prefectural Central Hospital, Houju Memorial Hospital, Komatsu Municipal Hospital, and Japan Community Healthcare Organization Kanazawa Hospital (approval no. 507, 14-2, 10 and 13-17-00, respectively). Patients who had received peripheral venous administration of L-OHP during April 2011 to March 2014 were collected from 5 hospitals in Ishikawa prefecture. We excluded patients who were fitted with a peripheral venous catheter port, or for whom any of the clinical data required in the study protocol were missing.

\section{Study protocol}

Data were collected from the nursing records and computerized medical records. Clinical data included age, gender, previous history of chemotherapy, dosage and infusion time of L-OHP, development of venous pain, concomitant drug used for analgesia, addition of dexamethasone, volume of infusion solution, and use of hot gel. For patients with a history of L-OHP-induced vascular pain, data were selected for the first cycle in which vascular pain occurred, while for patients who did not encounter vascular pain in any cycle, we selected data from the first cycle of L-OHP.
We assessed the incidence of L-OHP-induced vascular pain in patients classified according to use of analgesics, addition of dexamethasone, volume of infusion solution, or use of hot gel to warm the injection site, compared to patients who did not receive that treatment. Univariate analysis and multiple logistic regression analysis were conducted to identify independent risk factors associated with L-OHP-induced vascular pain. Based on the multiple logistic regression analysis, we also examined the relationship between the number of risk factors and the incidence of L-OHP-induced vascular pain.

\section{Statistical analyses}

The incidence of L-OHP-induced vascular pain in patients who received each preventive therapy was compared with the incidence in those who did not receive it by applying Fisher's exact test. The relationship between the number of risk factors and the incidence of L-OHP-induced vascular pain was also analyzed by means of Fisher's exact test. To identify risk factors associated with L-OHP-induced vascular pain, multiple logistic regression analysis was performed. Factors for which $P<0.20$ in univariate analysis were selected for multiple logistic regression analysis.

Data were analyzed using IBM SPSS Version 19 and $P$ values of $<0.05$ were considered statistically significant.

\section{Results}

\section{Patient characteristics}

One hundred and ninety colorectal cancer patients who had received peripheral venous administration of L-OHP were studied. The total number of infusions of L-OHP was 1,264 . There were 117 males and 73 female with a mean age of $64.2 \pm 11.3$ years (Table 1). The dosage of dexamethasone mixed with infusion solution for prevention of vascular pain was $1.65 \mathrm{mg}$ in all patients who received this preventive method. Use of analgesic was defined as routine administration of nonsteroidal anti-inflammatory drugs (NSAIDs) and/or opioids. NSAIDs administered to patients in this study were loxoprofen, diclofenac, acetaminophen, and celecoxib, and opioids used in this study were morphine sulfate, oxycodone hydrochloride and fentanyl patch. As the number of patients given analgesics was very small (Table 2), the preventive effect on vascular pain could not be analyzed precisely.

Incidence of L-OHP-induced vascular pain in patients with or without use of analgesics, addition with dexamethasone, dilution of infusion solution, or use of hot gel

The incidences of L-OHP-induced vascular pain were $56.3 \%$ in patients receiving analgesics, $78.3 \%$ in those given dexamethasone, $67.2 \%$ in those receiving diluted 
Table 1 Patient characteristics

\begin{tabular}{|c|c|c|c|}
\hline Number of patients (number) & & & 190 \\
\hline Number of infusions (number) & & & 1264 \\
\hline \multicolumn{4}{|l|}{ Gender } \\
\hline & & Male & 117 \\
\hline & & Female & 73 \\
\hline Age (year) & means $\pm S D$ & & $64.2 \pm 11.3$ \\
\hline BMI $\left(\mathrm{kg} / \mathrm{m}^{2}\right)$ & means $\pm S D$ & & $22.1 \pm 3.2$ \\
\hline \multicolumn{4}{|c|}{ History of chemotherapy (number) } \\
\hline & & $1 s t$ & 173 \\
\hline & & 2nd & 17 \\
\hline \multicolumn{4}{|l|}{ Clinical stage (number) } \\
\hline & & । & 2 \\
\hline & & $\|$ & 15 \\
\hline & & III & 92 \\
\hline & & IV & 81 \\
\hline \multicolumn{4}{|l|}{ L-OHP dosage (number) } \\
\hline & & $130 \mathrm{mg} / \mathrm{m}^{2}$ & 92 \\
\hline & & $<130 \mathrm{mg} / \mathrm{m}^{2}$ & 98 \\
\hline
\end{tabular}

$S D$ standard deviation

infusion solution and $63.3 \%$ in those who were given hot gel to warm the injection site. None of these methods had a significant benefit in terms of reducing the incidence of vascular pain (Fig. 1).

Univariate and multivariable analyses of risk factors for LOHP-induced vascular pain

In univariate analysis, the factors with $P$ values $<0.20$ were BMI, clinical stage, history of chemotherapy, and L-OHP dosage (Table 2). These factors were included in the multivariable analysis, which indicated that BMI, clinical stage and L-OHP dosage were independent risk factors (Table 3).

\section{Relationship between number of risk factors and incidence of L-OHP-induced vascular pain}

The incidences of L-OHP-induced vascular pain were $41.2 \%$ in patients who had no risk factor, $52.0 \%$ in those who had one risk factor and $79.6 \%$ in those who had two or more risk factors. Patients with two or more risk factors showed significantly increased incidence of vascular pain compared to those without risk factors (Fig. 2).

\section{Discussion}

In this study, we identified BMI $(\mathrm{BMI}<22)$, clinical stage (I-III) and L-OHP dosage $\left(130 \mathrm{mg} / \mathrm{m}^{2}\right.$ versus dose reduction) as independent risk factors of L-OHP-induced vascular pain. Generally, treatment to prevent vascular pain is given to patients who have already experienced vascular pain during a previous administration. Our results suggest that patients with two or more two risk factors might be at particular risk of vascular pain even in the first course.

Unfortunately, all the preventive methods examined in this study, including addition of dexamethasone to adjust the $\mathrm{pH}$ of the infusion solution and dilution of infusion solution, proved to be ineffective in reducing the incidence of vascular pain (Fig. 1). Although the $\mathrm{pH}$ or osmotic pressure of the infusion solution was proposed to cause vascular pain, our results indicate that this is unlikely. The $\mathrm{pH}$ of infusion solution containing $130 \mathrm{mg} / \mathrm{m}^{2} \mathrm{~L}-\mathrm{OHP}$ in Japanese patients of average physique was 4.81 after dilution with $250 \mathrm{~mL}$ of $5 \%$ glucose solution and 4.82 after dilution with $500 \mathrm{~mL}$. Addition of $3.3 \mathrm{mg}$ dexamethasone elevated the $\mathrm{pH}$ of L-OHP infusion solution to 7.18 in $250 \mathrm{~mL}$ of $5 \%$ glucose solution and to 6.84 in $500 \mathrm{~mL}$ [14]. Patients in this study received $1.65 \mathrm{mg}$ of dexamethasone, which should have been sufficient to elevate the $\mathrm{pH}$ of the L-OHP infusion solution, but the incidence of L-OHP-induced vascular pain was not reduced. On the other hand, the osmotic pressure of L-OHP infusion solution was $318 \mathrm{mEq} / \mathrm{L}$ after dilution with $250 \mathrm{~mL}$ of $5 \%$ glucose solution and $307 \mathrm{mEq} / \mathrm{L}$ after dilution with $500 \mathrm{~mL}$ [14], while the normal plasma osmotic pressure is approximately $300 \mathrm{mEq} / \mathrm{L}$. These results suggest that neither $\mathrm{pH}$ nor osmotic pressure of the L-OHP infusion solution was related to the induction of vascular pain. It was reported that addition of dexamethasone to L-OHP infusion solution and heating the infusion site had a preventive or ameliorating effect on vascular pain $[9,10]$. In this study, however, we assessed only whether or not the treatments altered the incidence of vascular pain, as the available data did not allow us to determine whether they were effective in reducing the severity of pain. Nagao et al. have reported that pretreatment with fast-acting oxycodone reduced vascular pain induced by oxaliplatin via a peripheral vein [15]. However, in our study, treatment with analgesics including opioids and/or NSAIDs did not reduce the incidence of vascular pain (Fig. 1). One of the differences between the two studies was the administration time of analgesics. Nagao et al. administered fast-acting oxycodone to patients $45 \mathrm{~min}$ before the administration of L-OHP. On the other hand, since the purpose of analgesics, including opioids and NSAIDs, in the patients in our study was to relieve cancer-related pain, the administration times might not have been appropriate to prevent vascular pain, resulting in the apparent ineffectiveness of analgesics. Carefully timed pretreatment with fast-acting oxycodone could be a useful option for management of L-OHP-induced vascular pain.

We identified three independent risk factors for L-OHP-induced vascular pain: L-OHP dosage, BMI and clinical stage (Table 3). Takagi et al. have already reported that L-OHP dosages of more than $175 \mathrm{mg} /$ body 
Table 2 Univariate analysis of risk factors for oxaliplatin-induced vascular pain

\begin{tabular}{|c|c|c|c|c|c|c|}
\hline & & Vascular pain (+) & Vascular pain $(-)$ & Odds ratio & $95 \% \mathrm{Cl}$ & $P$ value \\
\hline & Incidence (\%) & $n=124$ & $n=66$ & & & \\
\hline \multicolumn{7}{|l|}{ Gender } \\
\hline Female & 64.4 & 47 & 26 & 0.94 & $0.51-1.73$ & 0.876 \\
\hline Male & 65.8 & 77 & 40 & & & \\
\hline \multicolumn{7}{|l|}{ Age } \\
\hline$\geqq 70$ & 62.1 & 41 & 25 & 0.81 & $0.43-1.51$ & 0.526 \\
\hline$<70$ & 66.9 & 83 & 41 & & & \\
\hline \multicolumn{7}{|l|}{ BMI } \\
\hline$\geqq 22$ & 58.2 & 53 & 38 & 0.55 & $0.30-1.01$ & 0.067 \\
\hline$<22$ & 71.7 & 71 & 28 & & & \\
\hline \multicolumn{7}{|l|}{ Clinical stage } \\
\hline IV & 56.8 & 46 & 35 & 0.52 & $0.29-0.96$ & 0.045 \\
\hline$|-|||$ & 71.6 & 78 & 31 & & & \\
\hline \multicolumn{7}{|c|}{ History of chemotherapy } \\
\hline 2nd & 41.2 & 7 & 10 & 0.34 & $0.12-0.93$ & 0.035 \\
\hline $1 \mathrm{st}$ & 67.6 & 117 & 56 & & & \\
\hline \multicolumn{7}{|l|}{ L-OHP dosage } \\
\hline$<130 \mathrm{mg} / \mathrm{m}^{2}$ & 57.1 & 56 & 42 & 0.47 & $0.25-0.87$ & 0.022 \\
\hline $130 \mathrm{mg} / \mathrm{m}^{2}$ & 73.9 & 68 & 24 & & & \\
\hline \multicolumn{7}{|c|}{ Solution volume mixed with oxaliplatin } \\
\hline $500 \mathrm{~mL}$ & 67.2 & 41 & 20 & 1.14 & $0.60-2.17$ & 0.746 \\
\hline $250 \mathrm{~mL}$ & 64.3 & 83 & 46 & & & \\
\hline \multicolumn{7}{|c|}{ Warming the injection site } \\
\hline Yes & 63.3 & 81 & 47 & 0.76 & $0.40-1.46$ & 0.516 \\
\hline No & 69.4 & 43 & 19 & & & \\
\hline \multicolumn{7}{|l|}{ Analgesics } \\
\hline Yes & 56.3 & 9 & 7 & 0.66 & $0.23-1.86$ & 0.425 \\
\hline No & 66.1 & 115 & 59 & & & \\
\hline \multicolumn{7}{|l|}{ Opioids } \\
\hline Yes & 62.5 & 5 & 3 & 0.88 & $0.20-3.81$ & 1.000 \\
\hline No & 65.4 & 119 & 63 & & & \\
\hline \multicolumn{7}{|l|}{ Non-opioids } \\
\hline Yes & 54.5 & 6 & 5 & 0.62 & $0.18-2.12$ & 0.519 \\
\hline No & 65.9 & 118 & 61 & & & \\
\hline \multicolumn{7}{|c|}{ pH adjustment (addition of dexamethasone to infusion solution mixed with oxaliplatin) } \\
\hline Yes & 78.3 & 18 & 5 & 2.07 & $0.73-5.86$ & 0.242 \\
\hline No & 63.5 & 106 & 61 & & & \\
\hline
\end{tabular}

Cl confidence interval

are significantly related the development of vascular pain [16], suggesting that vascular pain is more likely to occur at higher L-OHP dosages. In general, L-OHP is administered over a period of $2 \mathrm{~h}$. Lengthening the administration time of L-OHP would reduce the L-OHP exposure per hour, and thus might be effective to prevent vascular pain. However, it would be necessary to confirm that this does not affect the efficacy or safety of L-OHP-containing regimens. Our multivariable analysis also indicated that clinical stage I-III and $\mathrm{BMI}<22$ were independent risk factors for vascular pain. However, although vascular pain, clinical stage and BMI were all identified as risk factors by multivariate analysis, there is no clear evidence to indicate the nature of the 

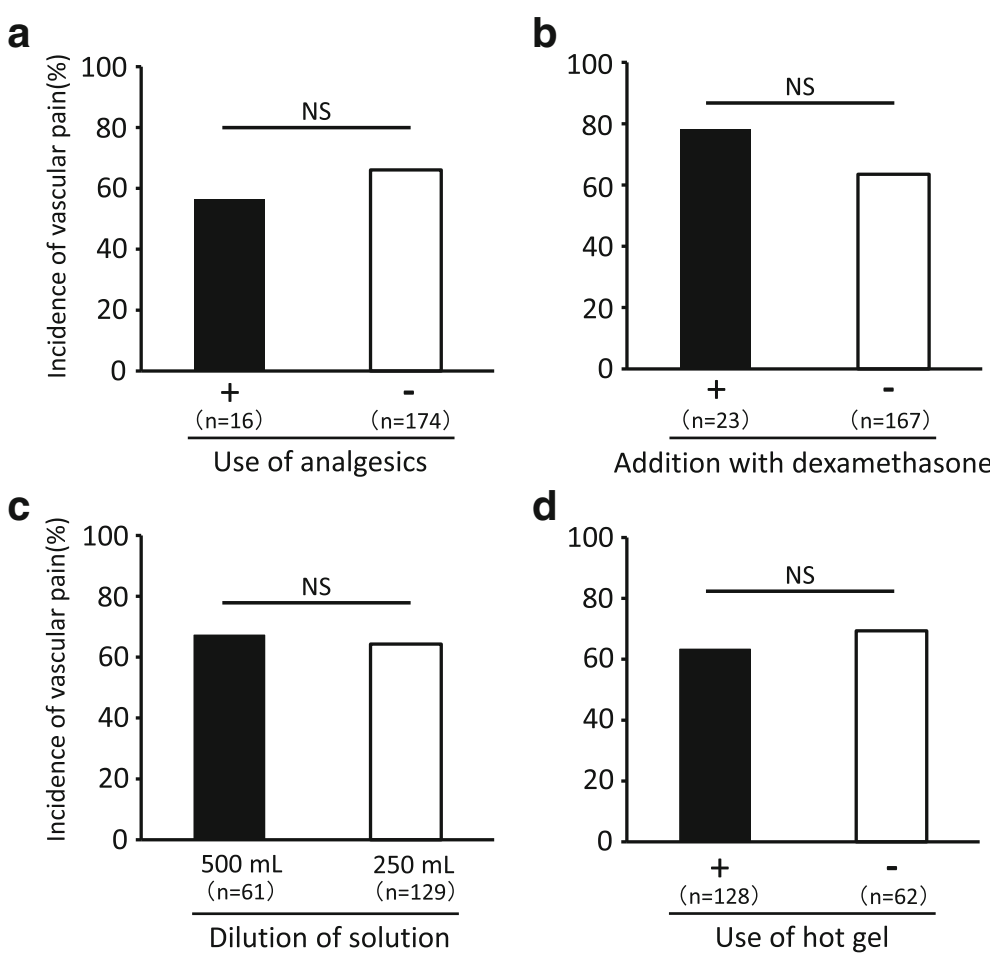

Fig. 1 The incidence of L-OHP-induced vascular pain in patients with or without use of analgesics (a), addition of dexamethasone to adjust the $\mathrm{pH}$ of the infusion solution (b), dilution of the infusion solution (c) and use of hot gel to warm the injection site (d). NS: not significant (Fischer's exact test)

relationship among them, or what the underlying mechanism might be. There is a report that acute cold sensitivity is one of the symptoms of acute peripheral neuropathy induced by L-OHP [5]. The acute cold sensitivity appeared within a few hours after starting infusion of oxaliplatin, at the same time as vascular pain, so a similar mechanism might be involved. Transient receptor potential ankyrin 1

Table 3 Multivariate analysis of risk factors for oxaliplatininduced vascular pain

\begin{tabular}{|c|c|c|c|}
\hline & Adjusted odds ratio & $95 \% \mathrm{Cl}$ & $P$ value \\
\hline \multicolumn{4}{|l|}{ BMI } \\
\hline$\geqq 22$ & 0.48 & $0.26-0.91$ & 0.025 \\
\hline \multicolumn{4}{|l|}{$<22$} \\
\hline \multicolumn{4}{|l|}{ Clinical stage } \\
\hline IV & 0.52 & $0.27-0.97$ & 0.041 \\
\hline \multicolumn{4}{|l|}{$|-|||$} \\
\hline \multicolumn{4}{|c|}{ History of chemotherapy } \\
\hline $2 n d$ & 0.43 & $0.15-1.28$ & 0.129 \\
\hline \multicolumn{4}{|l|}{$1 \mathrm{st}$} \\
\hline \multicolumn{4}{|l|}{ L-OHP dosage } \\
\hline$<130 \mathrm{mg} / \mathrm{m}^{2}$ & 0.51 & $0.27-0.98$ & 0.042 \\
\hline $130 \mathrm{mg} / \mathrm{m}^{2}$ & & & \\
\hline
\end{tabular}

(TRPA1) is activated by noxious cold stimulation and various irritants, such as reactive oxygen species (ROS) [17], and is involved in acute cold sensitivity in mice [18]. On the other hand, Ando et al. reported that TRPA1 was related to the development of vascular pain induced by propofol in a rat model [19]. However, it is not known whether or not TRPA1 is related to L-OHP-induced

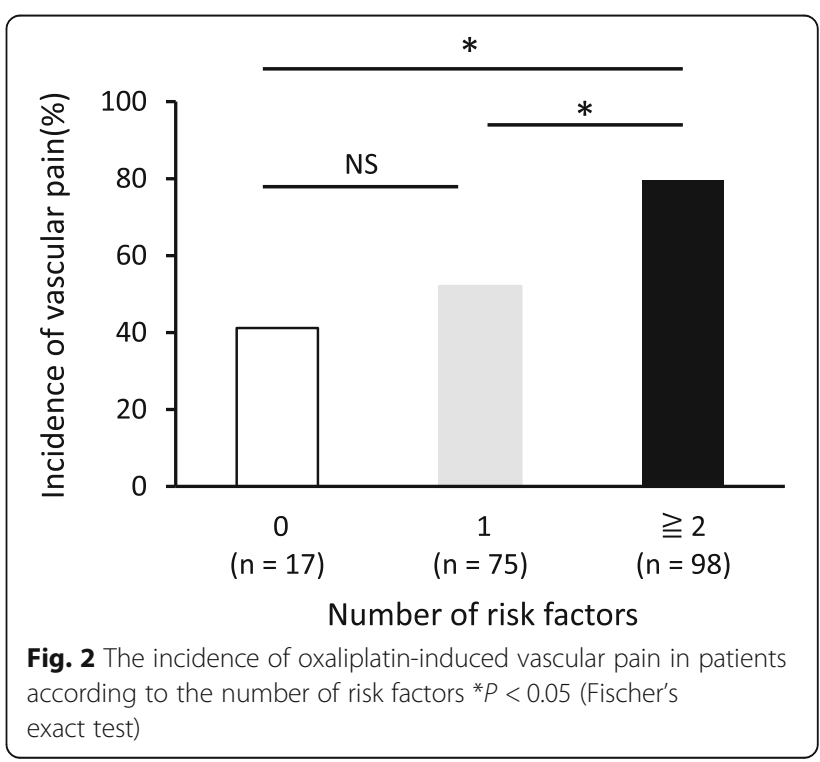


vascular pain. Further research will be needed to elucidate this point and to uncover the relationship between vascular pain and BMI or clinical stage.

Our results indicate that BMI, clinical stage and L-OHP dosage are useful predictive markers for L-OHP-induced vascular pain in patients with colorectal cancer. None of the preventive methods examined was effective in reducing the incidence of vascular pain, but we could not establish whether they reduced the degree of pain. Since the incidence of vascular pain was higher in patients with two or more risk factors, it may therefore be worthwhile to administer preventive treatment to high-risk individuals even upon first administration of L-OHP. Further studies are needed to elucidate the mechanism of vascular pain in order to develop effective preventive methods, as well as to compare the efficacy of the various existing treatments in ameliorating pain.

\section{Conclusions}

The results of this retrospective study indicate that L-OHP dose (130 $\mathrm{mg} / \mathrm{m}^{2}$ versus dose reduction), clinical stage I-III and $\mathrm{BMI}<22$ are risk factors for L-OHP-induced vascular pain in patients with colorectal cancer. These findings could be useful to assess the risk of vascular pain in clinical settings. Based on the results of this study together with previous findings, we suggest that the best option for management of L-OHP-induced vascular pain may be carefully timed pretreatment with fast-acting oxycodone.

\section{Acknowledgements}

We would like to thank the following pharmacists for collecting clinical data; Chiyoko Yamada, Anna Kubo: Department of Pharmacy, Ishikawa Prefectural Central Hospital.

\section{Funding}

This work was supported by a Grant-in-Aid for Young Scientists (B) from the Japan Society for the Promotion of Science (Grant Number JP25860109).

\section{Availability of data and materials}

The dataset supporting the conclusions of this article is included within the article.

\section{Authors' contributions}

YuS conceived the study, designed the protocol, carried out the study and drafted the manuscript. NI, MM, AS, MY, HK, HK, MO collected a data from clinical record in each institutions. TS, Jl, YoS, and RM coordinated the study and helped to draft the manuscript. YuS wrote the paper. All authors revised the manuscript for intellectual content and approved the final manuscript.

\section{Ethics approval and consent to participate}

Protocol of this study was approved by the Ethics Committee of Kanazawa University (approval no. 1462), as well as by the ethics committees of all participating hospitals, which were Ishikawa Prefectural Central Hospital, Houju Memorial Hospital, Komatsu Municipal Hospital, and Japan Community Healthcare Organization Kanazawa Hospital (approval no. 507, $14-2,10$ and 13-17-00, respectively).

\section{Consent for publication}

Not applicable.

\section{Competing interests}

The authors declare that they have no conpeting interest.

\section{Publisher's Note}

Springer Nature remains neutral with regard to jurisdictional claims in published maps and institutional affiliations.

\section{Author details}

${ }^{1}$ Department of Clinical Drug Informatics, Faculty of Pharmacy, Institute of Medical, Pharmaceutical \& Health Science, Kanazawa University, 13-1, Takaramachi, Kanazawa, Ishikawa 920-8641, Japan. ²Department of Pharmacy, Kanazawa University Hospital, Kanazawa University, 13-1, Takaramachi, Kanazawa, Ishikawa 920-8641, Japan. ${ }^{3}$ Department of Pharmacy, Ishikawa Prefectural Central Hospital, 2-1, Kuratsukihigashi, Kanazawa, Ishikawa 920-8530, Japan. ${ }^{4}$ Department of Pharmacy, Houju Memorial Hospital, 11-71, Midorigaoka, Nomi, Ishikawa 923-1226, Japan. ${ }^{5}$ Department of Pharmacy, Komatsu Municipal Hospital, HO-60, Mukai Moto-ori-machi, Komatsu, Ishikawa 923-8560, Japan. 'Department of Pharmacy, Japan Community Healthcare Organization Kanazawa Hospital, Ha-15, Oki-machi, Kanazawa, Ishikawa 920-8610, Japan.

Received: 11 April 2018 Accepted: 2 July 2018

Published online: 07 August 2018

\section{References}

1. De Gramont A, Figer A, Seymour M, Homerin M, Hmissi A, Cassidy J, et al. Leucovorin and fluorouracil with or without oxaliplatin as first-line treatment in advanced colorectal cancer. J Clin Oncol. 2000;18:2938-47.

2. Andre T, Boni C, Mounedji-Boudiaf L, Navarro M, Tabernero J, Hickish T, et al. Oxaliplatin, fluorouracil, and leucovorin as adjuvant treatment for colon cancer. N Engl J Med. 2004;350:2343-51.

3. Cassidy J, Misset JL. Oxaliplatin-related side effects: characteristics and management. Semin Oncol. 2002;29:11-20.

4. Land SR, Kopec JA, Cecchini RS, Ganz PA, Wieand HS, Colangelo LH, et al. Neurotoxicity from oxaliplatin combined with weekly bolus fluorouracil and leucovorin as surgical adjuvant chemotherapy for stage II and III colon cancer: NSABP C-07. J Clin Oncol. 2007;25:2205-11.

5. Krishnan AV, Goldstein D, Friedlander M, Kiernan MC. Oxaliplatin-induced neurotoxicity and the development of neuropathy. Muscle Nerve. 2005;32: 51-60.

6. Picard P, Tramer MR. Prevention of pain on injection with propofol: a quantitative systematic review. Anesth Analg. 2000;90:963-9.

7. Ozgul U, Begec Z, Erdogan MA, Aydogan MS, Sanli M, Colak C. Effect of alkalinisation of lignocaine for propofol injection pain: a prospective, randomised, double-blind study. Anaesth Intensive Care. 2013:41:501-4.

8. Chaudhary K, Gupta P, Gogia AR. A prospective, randomized, double-blind study to compare the efficacy of lidocaine + metoclopramide and lidocaine + ketamine combinations in preventing pain on propofol injection. J Anesth. 2013;27:402-6.

9. Yatate M, Kimura M, Iwasaki Y, Kamura M, Yokoi M, Uchitani S, et al. Reduction of vascular pain due to drop-infusion of oxaliplatin (L-OHP) in peripheral vein with heated infusion route. Japan J Cancer Chemother. 2012;39:589-91.

10. Shiotsuka Y, Uebuchi M, Hamada S, Morita N, Moriyama A, Yamashita M, et al. Reduction of angialgia during peripheral administration of oxaliplatin mixed with dexamethasone. Japan J Cancer Chemother. 2012;39:1583-6.

11. Hibi S, Tatematsu M, Nishio S, Miyatani M, Tosaki K, Ohara M, et al. Investigating the incidence of injection-site reactions associated with administration of oxaliplatin into a peripheral vein and management local adverse reactions. Japan J Cancer Chemother. 2011;38:1447-52.

12. Nagata K, Egashira N, Yamada T, Watanabe H, Yamauchi Y, Oishi R. Change of formulation decreases venous irritation in breast cancer patients receiving epirubicin. Support Care Cancer. 2012;20:951-5.

13. Suga $Y$, Kumazaki M, Nishigami J, Takeda K, Kawagishi A, Ishizaki J, et al. Improvement of epirubicin-induced phlebitis to switch from liquid preparation to lyophilized formulation. Japan J Cancer Chemother. 2009;36: 93-6.

14. Matsuyama K, Mishima H, Ueno H, Kajihara K, Morioka A, Morimoto S, et al. Etiology and management of venous pain during intravenous administration of oxaliplatin. Japan J Cancer Chemotherapy. 2011;38:411-4. 
15. Nagao S, Furihata M, Fukagawa K, Furihata T, Matsuhashi Y, Wada T. Premedication with fast-acting oxycodone hydrochloride hydrate effectively reduced oxaliplatin-induced severe vascular pain. J Infect Chemother. 2017; 23:493-7.

16. Takagi A, Yonemoto N, Aoyama Y, Touma Y, Kajiwara M, Watanabe K, et al. Search for factors related to vascular pain expression upon administration of oxaliplatin into a peripheral vein. Japan J Cancer Chemother. 2015;42:887-9.

17. Miyake T, Nakamura S, Zhao M, So K, Inoue K, Numata T, et al. Cold sensitivity of TRPA1 is unveiled by the prolylhydroxylation blockade-induced sensitization to ROS. Nat Commun 2016; doi: https://doi.org/10.1038/ ncomms 12840.

18. Zhao M, Isami K, Nakamura S, Shirakawa H, Nakagawa T, Kaneko S. Acute cold hypersensitivity characterstically induced by oxaliplatin is caused by the enhanced responsiveness of TRPA1 in mice. Mol Pain 2012; doi: https:// doi.org/10.1186/1744-8069-8-5.

19. Ando R, Watanabe $C$. Characteristics of propofol-evoked vascular pain in anaesthetized rats. Br J Anaesth. 2005;95:384-92.

Ready to submit your research? Choose BMC and benefit from:

- fast, convenient online submission

- thorough peer review by experienced researchers in your field

- rapid publication on acceptance

- support for research data, including large and complex data types

- gold Open Access which fosters wider collaboration and increased citations

- maximum visibility for your research: over $100 \mathrm{M}$ website views per year 